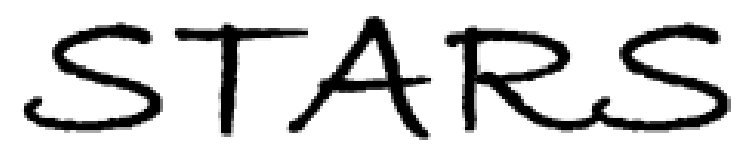

University of Central Florida

STARS

$1-1-2009$

\title{
Observation of temperature-independent internal Er3+ relaxation efficiency in Si-rich SiO2 films
}

Oleksandr Savchyn

University of Central Florida

Ravi M. Todi

University of Central Florida

Kevin R. Coffey

University of Central Florida

Pieter G. Kik

University of Central Florida

Find similar works at: https://stars.library.ucf.edu/facultybib2000

University of Central Florida Libraries http://library.ucf.edu

This Article is brought to you for free and open access by the Faculty Bibliography at STARS. It has been accepted for inclusion in Faculty Bibliography 2000s by an authorized administrator of STARS. For more information, please contactSTARS@ucf.edu.

\section{Recommended Citation}

Savchyn, Oleksandr; Todi, Ravi M.; Coffey, Kevin R.; and Kik, Pieter G., "Observation of temperatureindependent internal Er3+ relaxation efficiency in Si-rich SiO2 films" (2009). Faculty Bibliography 2000 s. 2094.

https://stars.library.ucf.edu/facultybib2000/2094

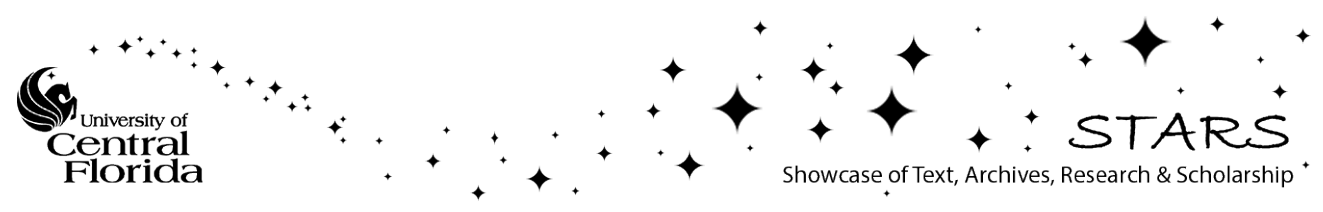




\section{Observation of temperature-independent internal $\mathrm{Er}^{3+}$ relaxation efficiency in Si-rich $\mathrm{SiO}_{2}$ films}

Cite as: Appl. Phys. Lett. 94, 241115 (2009); https://doi.org/10.1063/1.3157135

Submitted: 20 February 2009 . Accepted: 30 May 2009 . Published Online: 18 June 2009

Oleksandr Savchyn, Ravi M. Todi, Kevin R. Coffey, and Pieter C. Kik

\section{ARTICLES YOU MAY BE INTERESTED IN}

Multilevel sensitization of $\mathrm{Er}^{3+}$ in low-temperature-annealed silicon-rich $\mathrm{SiO}_{2}$ Applied Physics Letters 93, 233120 (2008); https://doi.org/10.1063/1.3044480

Coexistence of two different energy transfer processes in $\mathrm{SiO}_{2}$ films containing $\mathrm{Si}$ nanocrystals and Er

Journal of Applied Physics 95, 272 (2004); https://doi.org/10.1063/1.1631072

Resonant excitation of $\mathrm{Er}^{3+}$ by the energy transfer from Si nanocrystals

Journal of Applied Physics 90, 4761 (2001); https://doi.org/10.1063/1.1409572

\section{Applied Physics Letters}

Mid-IR and THz frequency combs special collection

Read Now! 


\title{
Observation of temperature-independent internal $\mathrm{Er}^{3+}$ relaxation efficiency in Si-rich $\mathrm{SiO}_{2}$ films
}

\author{
Oleksandr Savchyn, ${ }^{1, a)}$ Ravi M. Todi, ${ }^{2}$ Kevin R. Coffey, ${ }^{2, b)}$ and Pieter G. Kik ${ }^{1, b)}$ \\ ${ }^{1}$ CREOL, The College of Optics and Photonics, University of Central Florida, 4000 Central Florida Blvd., \\ Orlando, Florida 32816, USA \\ ${ }^{2}$ Advanced Materials Processing and Analysis Center (AMPAC), University of Central Florida, \\ 4000 Central Florida Blvd., Orlando, Florida 32816, USA
}

(Received 20 February 2009; accepted 30 May 2009; published online 18 June 2009)

\begin{abstract}
Time-dependent photoluminescence measurements of low-temperature-annealed Er-doped Si-rich $\mathrm{SiO}_{2}$ were conducted at sample temperatures 15-300 K. The erbium internal relaxation efficiency from the second $\left({ }^{4} I_{11 / 2}\right)$ to the first $\left({ }^{4} I_{13 / 2}\right)$ excited state upon luminescence-center-mediated $\mathrm{Er}^{3+}$ excitation is investigated. Despite the observation of temperature-dependent relaxation rates, the erbium internal relaxation efficiency is found to be remarkably temperature independent, which suggests that the internal relaxation efficiency is near unity. Internal relaxation is shown to account for $50 \%-55 \%$ of the ${ }^{4} I_{13 / 2}$ excitation events in the entire temperature range. These results demonstrate that high pump efficiency and stable operation of devices based on this material will be possible under varying thermal conditions. (C) 2009 American Institute of Physics.
\end{abstract}

[DOI: 10.1063/1.3157135]

Currently a significant amount of work is dedicated to the realization of a silicon-based light source for optical onchip interconnects. ${ }^{1-5}$ The recent discovery of luminescencecenter-mediated sensitization of $\mathrm{Er}^{3+}$ in Si-rich $\mathrm{SiO}_{2}{ }^{6,7}$ and the presence of $\mathrm{Er}^{3+}$ sensitization even in samples containing excess silicon but no $\mathrm{Si}$ nanocrystals $(\mathrm{NCs})^{6-8}$ open up new opportunities to achieve this goal. The use of Er-doped Sirich $\mathrm{SiO}_{2}$ without $\mathrm{Si}$ NCs could potentially overcome several challenges, including free carrier absorption, ${ }^{9,10}$ NC-related scattering losses, ${ }^{11}$ and the low density of sensitized $\mathrm{Er}^{3+}$ ions ${ }^{6}$ inherent to Er-doped Si-rich $\mathrm{SiO}_{2}$ with $\mathrm{Si}$ NCs. In recent study ${ }^{12}$ we demonstrated that the excitation of $\mathrm{Er}^{3+}$ in Si-rich $\mathrm{SiO}_{2}$ without $\mathrm{Si}$ NCs likely occurs not only into higher energy levels but also directly into the first excited state $\left({ }^{4} I_{13 / 2}\right)$. As a result, the first excited state of $\mathrm{Er}^{3+}$ is populated via two channels: through direct excitation by $\mathrm{Si}-$ related luminescence centers (LCs), and through LC-related excitation into higher lying levels followed by internal relaxation of the $\mathrm{Er}^{3+}$ ions to the first excited state. These two excitation pathways account for an approximately equal number of excitation events at $T=15 \mathrm{~K}^{12}$ In the current study we monitor temperature-dependent excitation and relaxation rates of $\mathrm{Er}^{3+}$ and demonstrate that the internal relaxation remains an efficient excitation channel up to room temperature.

Er-doped Si-rich $\mathrm{SiO}_{2}$ films containing 12 at. \% excess $\mathrm{Si}$ and 0.63 at. \% of Er were prepared by magnetron cosputtering onto a $\mathrm{Si}$ substrate. The samples were annealed for $100 \mathrm{~s}$ in $\mathrm{N}_{2}$ at $600{ }^{\circ} \mathrm{C}$ or $1100{ }^{\circ} \mathrm{C}$ (labeled LTA and HTA, respectively, for low and high temperature annealing) and subsequently passivated for $30 \mathrm{~min}$ in forming gas $\left(\mathrm{N}_{2}: \mathrm{H}_{2}\right.$ $=95 \%: 5 \%$ ) at $500{ }^{\circ} \mathrm{C}$. No Si aggregates could be detected in transmission electron microscopy measurements on LTA samples, while measurements on HTA samples clearly showed the presence of NCs. ${ }^{6}$ Optical measurements were performed in a closed-loop He cryostat in the temperature

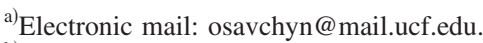

${ }^{b)}$ Also at Physics Department, University of Central Florida.
}

range 15-300 K. Photoluminescence (PL) spectra were taken under $351 \mathrm{~nm}$ excitation with a Kr-ion laser (pump irradiance $1.40 \mathrm{~W} / \mathrm{cm}^{2}$ ). The PL spectra were corrected for the system spectral response. The time-dependent PL signal was measured either under modulated continuous wave (cw) pumping using the excitation parameters described above or using pulsed excitation with the $355 \mathrm{~nm}$ line of a neodymium doped yttrium aluminum garnet laser with a pulse width of $5 \mathrm{~ns}$ and a pulse energy of $1.9 \mu \mathrm{J}$. All measured PL parameters have an experimental error smaller than $\pm 5 \%$. A more detailed description of the experimental procedures can be found in Refs. 6, 7, and 12.

The PL spectra of the LTA sample shown in Fig. 1(a) exhibit four emission bands: a band peaking at $\sim 600 \mathrm{~nm}$ corresponding to the emission from silicon-excess-related $\mathrm{LCs},{ }^{13}$ a band peaking at $1128 \mathrm{~nm}$ most likely corresponding to radiative exciton recombination in the $\mathrm{Si}$ substrate, and two Er-related emission bands at 981 and $1535 \mathrm{~nm}$ corresponding to the transitions ${ }^{4} I_{11 / 2} \rightarrow{ }^{4} I_{15 / 2}$ and ${ }^{4} I_{13 / 2} \rightarrow{ }^{4} I_{15 / 2}$, respectively. The PL spectra of the HTA sample shown in Fig. 1(b) exhibit three emission bands: a broad emission band peaking at $\sim 750 \mathrm{~nm}$ typically attributed to emission from $\mathrm{Si} \mathrm{NCs}$ in the Si-rich $\mathrm{SiO}_{2}$ matrix and the two Errelated emission bands that were also observed in the LTA sample. In both samples the Er-related emission at $1535 \mathrm{~nm}$ shows pronounced spectral broadening with increasing temperature due to the temperature-dependent redistribution of the excited states over the Stark-split ${ }^{4} I_{13 / 2}$ level. No $\mathrm{Er}^{3+}$ emission could be detected in samples containing a similar Er concentration $(0.49$ at. \%) but no $\mathrm{Si}$ excess under cw or pulsed excitation. This clearly demonstrates that the excitation of $\mathrm{Er}^{3+}$ in the LTA and HTA samples is predominantly indirect and $\mathrm{Si}$-excess related. To ensure similar excitation conditions the measurements under both $\mathrm{cw}$ and pulsed excitation were taken at sufficiently low pump power to avoid significant signal saturation and second order processes (cooperative upconversion, excited state absorption).

Based on lifetime measurements at $1535 \mathrm{~nm}$ under modulated $\mathrm{cw}$ excitation (not shown) the excitation rate of 


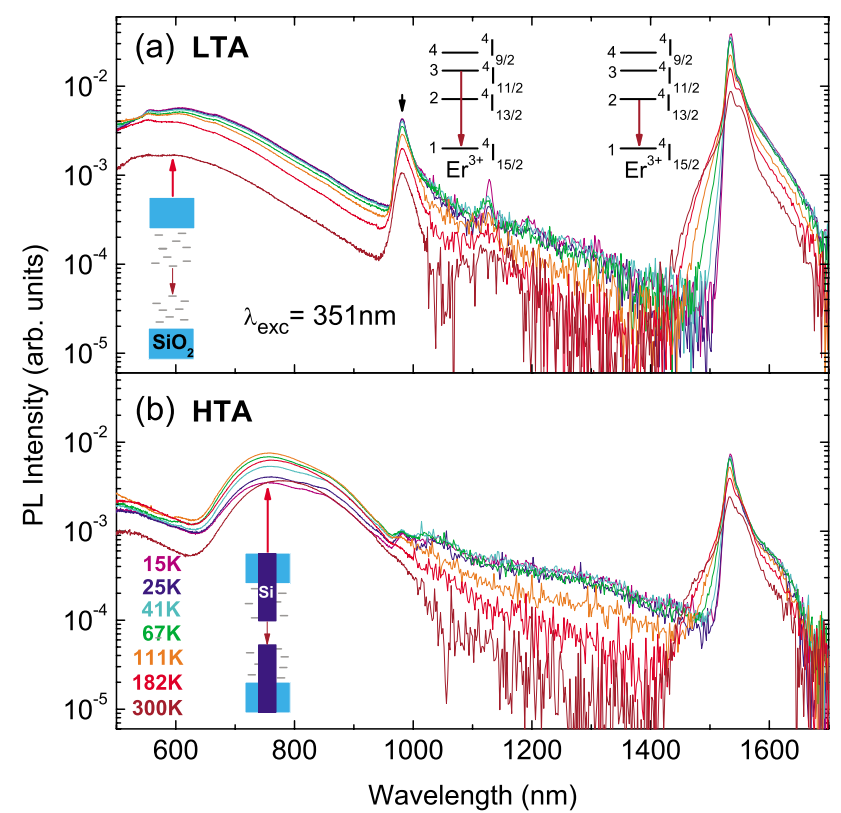

FIG. 1. (Color online) Temperature-dependent photoluminescence spectra of Er-doped Si-rich $\mathrm{SiO}_{2}$ annealed at $600{ }^{\circ} \mathrm{C}$ (LTA) (a) and at $1100{ }^{\circ} \mathrm{C}$ (HTA) (b). The corresponding optical transitions are schematically indicated.

the first excited state of $\mathrm{Er}^{3+}\left(R_{\text {exc }}\right)$ in LTA and HTA samples was determined using the well known relation $R_{\text {exc }}=\tau_{\text {rise }}^{-1}$ $-\tau_{\text {dec }}^{-1}$ where $\tau_{\text {rise }}$ and $\tau_{\text {dec }}$ are, respectively, the rise and decay times of the emission. The results are shown in Fig. 2(a). Note that despite the significant difference in the stoichiometry of the LTA and HTA samples, the observed excitation rates are similar in value and are both virtually temperature independent. This provides further evidence for a similar origin of $\mathrm{Er}^{3+}$ sensitization in these different samples.

Figures 3(a) and 3(b) show the time-dependent PL intensity at $981 \mathrm{~nm}$ in the LTA sample taken under pulsed excitation at $355 \mathrm{~nm}$. The excitation of the second excited state was found to take place on a time scale $<27 \mathrm{~ns}$ at

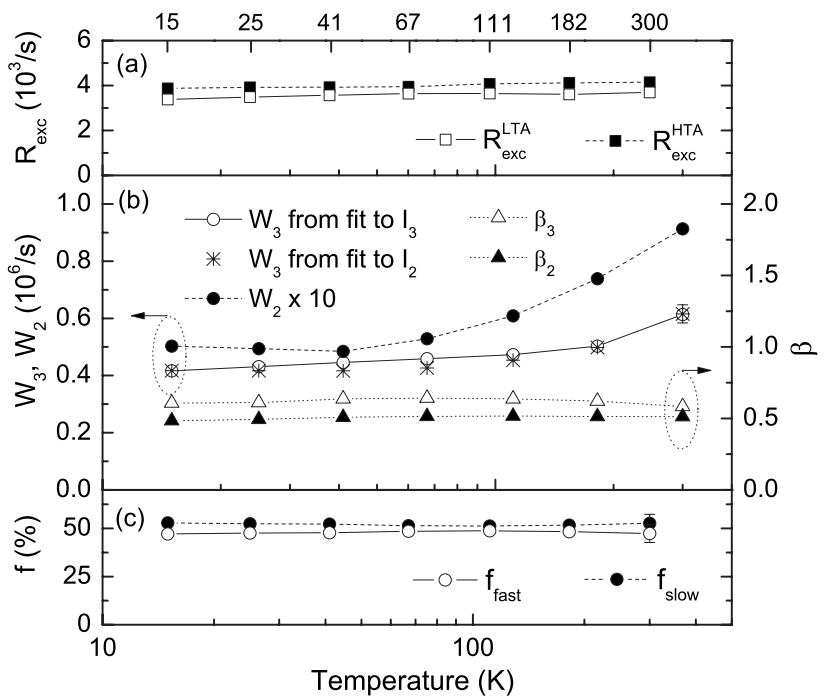

FIG. 2. Temperature dependence of (a) the $\mathrm{Er}^{3+}$ first excited state excitation rate in LTA $\left(R_{\mathrm{exc}}^{\mathrm{LTA}}\right)$ and HTA $\left(R_{\mathrm{exc}}^{\mathrm{HTA}}\right)$ samples, $(\mathrm{b})$ the $\mathrm{Er}^{3+}$ second excited state relaxation rate $\left(W_{3}\right)$ found from the time-dependent intensities at 981 $\mathrm{nm}\left(I_{3}\right)$ and $1535 \mathrm{~nm}\left(I_{2}\right)$, the first excited state relaxation rate $\left(W_{2}\right)$, and the dispersion factors corresponding to the relaxation from the first $\left(\beta_{2}\right)$ and second $\left(\beta_{3}\right)$ excited states, (c) the relative fractions of the first excited state excitation events due to the fast $\left(f_{\text {fast }}\right)$ and slow $\left(f_{\text {slow }}\right)$ excitation processes.

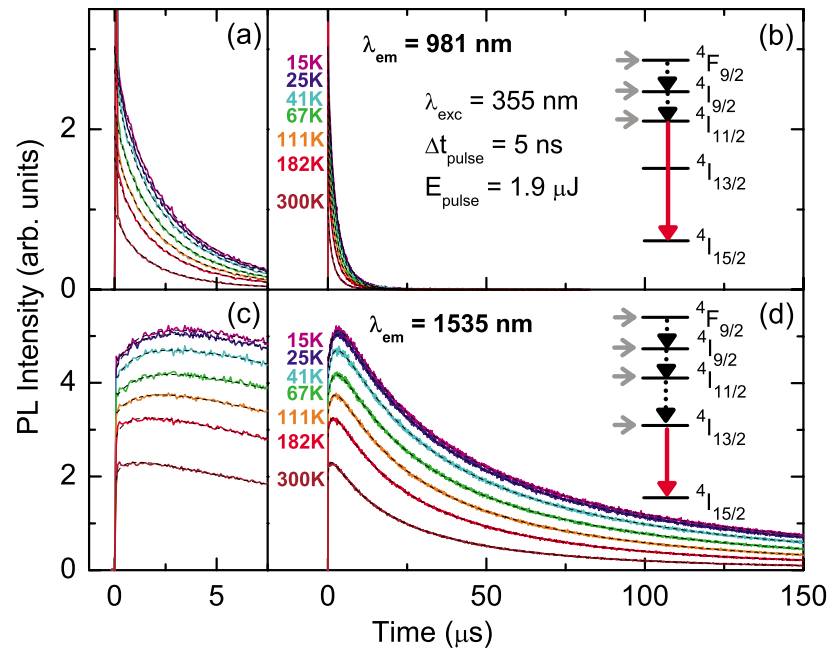

FIG. 3. (Color online) Time-dependent $\mathrm{Er}^{3+} \mathrm{PL}$ intensity at $981 \mathrm{~nm}[(\mathrm{a})$ and (b)] and at $1535 \mathrm{~nm}[(\mathrm{c})$ and (d)] under pulsed excitation at $355 \mathrm{~nm}$ (solid lines) with the corresponding fits (dashed lines). All intensities are shown on the same relative scale. Contributing excitation channels are schematically indicated by the horizontal and dashed vertical arrows. The relevant optical transitions are indicated by the solid vertical arrows.

all temperatures. The 1/e decay times of the second excited state of $\mathrm{Er}^{3+}\left({ }^{4} I_{11 / 2}\right) \tau_{3}$ were obtained by fitting the measured decay traces with the function $I_{3}(t)=I_{3}(0) \times \exp \left[-\left(t / \tau_{3}\right)^{\beta_{3}}\right]$. All decay traces exhibited a temperature-independent value of $\beta_{3}=0.79+/-0.01$. The corresponding decay rate of the second excited state of $\mathrm{Er}^{3+}$ in the LTA sample $W_{3}=1 / \tau_{3}$ is shown in Fig. 2(b) and demonstrates a gradual increase by a factor of $\sim 1.5$ as the temperature is increased from 15 to $300 \mathrm{~K}$.

Figures 3(c) and 3(d) show the time-dependent PL intensity at $1535 \mathrm{~nm}$ in the LTA sample taken under pulsed excitation. The shape of these traces results from the presence of two distinct sensitization mechanisms: ${ }^{12}$ a fast excitation process taking place on a timescale $<27$ ns due to excitation of $\mathrm{Er}^{3+}$ by LCs apparently directly into the first excited state and a slow excitation process taking place on a timescale of several microseconds due to the LC-mediated excitation of $\mathrm{Er}^{3+}$ into higher energy levels followed by relatively slow relaxation to the first excited state. The short time constant $(<27 \mathrm{~ns})$ of the fast excitation of both the first and second excited states at all considered temperatures suggests a similar origin of the fast excitation of both states. The experimental decay traces at $1535 \mathrm{~nm}$ are described by the function: ${ }^{12}$

$$
I_{2} \propto N_{2}^{\text {fast }}(0) e^{-\left(t / \tau_{2}\right)^{\beta_{2}}}+\frac{N_{3}(0)}{\tau_{32}\left(\tau_{3}^{-1}-\tau_{2}^{-1}\right)}\left[e^{-\left(t / \tau_{2}\right)^{\beta_{2}}}-e^{-\left(t / \tau_{3}\right)^{\beta_{3}}}\right],
$$

where $N_{2}^{\text {fast }}(0)$ and $N_{3}(0)$ are the densities of the $\mathrm{Er}^{3+}$ ions excited by the fast process into the first and higher excited states, respectively, $\tau_{2}, \beta_{2}$ and $\tau_{3}, \beta_{3}$ are the decay times and the dispersion factors, respectively, of the first and second excited states and $\tau_{32}$ is the relaxation time from the second to the first excited state of $\mathrm{Er}^{3+}$. The thus obtained $W_{3}$ $=1 / \tau_{3}$ values are in excellent agreement with the corresponding decay rates acquired from the fit to the time-dependent $981 \mathrm{~nm}$ emission [Fig. 2(b)], strongly supporting the presented model. The decay rate of the first excited state $\left(W_{2}\right.$ $=1 / \tau_{2}$ ) of the LTA sample is included in Fig. 2(b) and shows a similar to $W_{3}$ increase by a factor of $\sim 1.9$. The values of 
the dispersion factors $\beta_{2}$ and $\beta_{3}$ describe the observed multiexponential decay, which is likely caused by the presence of $\mathrm{Er}^{3+}$ ions with different decay rates. The obtained dispersion factors are shown in Fig. 2(b), and can be seen to be relatively constant, suggesting that temperature affects all $\mathrm{Er}^{3+}$ ions responsible for the emission in a similar manner.

The relaxation rate $W_{3}$ is commonly assumed to be dominated by multiphonon relaxation from the second to the first excited state, with the energy difference bridged predominantly by high energy phonons ${ }^{14}$ with energies of $\sim 130-150 \mathrm{meV}$ in the case of $\mathrm{SiO}_{2} \cdot{ }^{15,16}$ However, using the well known expression for the multiphonon relaxation rate, ${ }^{14}$ the temperature dependence of $W_{3}$ could not be accurately described. This suggests that a non-phonon-related temperature-dependent nonradiative decay channel is present. This assumption is supported by the room temperature relaxation rate of $W_{3}=6.2 \times 10^{5} \mathrm{~s}^{-1}$, which is much larger than both the room temperature multiphonon rate of $\sim 1 \times 10^{5} \mathrm{~s}^{-1}$ (Ref. 14) and the typical radiative rate of $\sim 100-130 \mathrm{~s}^{-1}$ (Ref. 17) of the second excited state of $\mathrm{Er}^{3+}$ in silicate glasses. Note that the first excited state decay rate $W_{2}$, which is known to be approximately temperature independent in $\mathrm{SiO}_{2}$ (Ref. 17) also increases by a factor of $\sim 1.9$ in this temperature range. Both observations are likely due to the presence of nonradiative relaxation through coupling with low-energy electronic transitions in this lowtemperature processed $\mathrm{Si}$-doped oxide. This suggests that the technical implementation of this material requires thermal processing that minimizes the presence of electronic defects, while avoiding the formation of extended Si NCs.

The temperature dependence of the fractions of $\mathrm{Er}^{3+}$ ions excited into the first excited state via the fast $\left(f_{\text {fast }}\right)$ and slow $\left(f_{\text {slow }}\right)$ process, respectively, found from the time integration of the first and second terms in Eq. (1) (Ref. 12) is shown in Fig. 2(c). Surprisingly, for all temperatures the fast and slow excitation mechanisms are responsible for $45 \%-50 \%$ and $50 \%-55 \%$, respectively, of the excitation events into the first excited state. Assuming that the fast excitation of the first and second excited states have the same temperature dependence, the observed temperature-independent contribution of each of the excitation pathways of the first excited state suggests that the relaxation efficiency from the second to the first excited state of $\mathrm{Er}^{3+}\left(\eta_{32}\right)$ does not change significantly with temperature. However, the total relaxation rate from the second excited state $\left(W_{3}\right)$ does vary with temperature by a factor of $\sim 1.5$, increasing from $4.2 \times 10^{5} \mathrm{~s}^{-1}$ at $15 \mathrm{~K}$ to $6.2 \times 10^{5} \mathrm{~s}^{-1}$ at room temperature [Fig. 2(b)]. Since $W_{3}$ $=W_{32}+W_{31}$ where $W_{32}=1 / \tau_{32}$ is the relaxation rate from the second to the first excited state and $W_{31}$ is the relaxation rate from the second excited state to the ground state, this increase in $W_{3}$ indicates that at least one of the two contributing relaxation rates $W_{32}$ and $W_{31}$ increases with temperature. This in turn would lead to a temperature-dependent relaxation quantum efficiency from the second to the first excited state given by $\eta_{32}=W_{32} / W_{3}=\left(1+W_{31} / W_{32}\right)^{-1}$, which would be accompanied by a change of the excitation rate of the first excited state $\left(R_{\text {exc }}\right)$, and a change of the relative fraction of $\mathrm{Er}^{3+}$ ions excited into the first excited state by the slow excitation mechanism $\left(f_{\text {slow }}\right)$. Based on the fact that neither of these changes are observed we conclude that the relaxation efficiency is temperature independent.

The temperature independent relaxation efficiency can be explained by assuming a relatively slow relaxation from the second excited state to the ground state $\left(W_{31} \ll W_{32}\right.$ and thus $\left.W_{3}=W_{32}+W_{31} \approx W_{32}\right)$ resulting in $W_{31} / W_{32} \ll 1$. Under this assumption and realizing that $\eta_{32}=W_{32} / W_{3}=(1$ $\left.+W_{31} / W_{32}\right)^{-1}$ we find $\eta_{32} \approx 1$. Alternatively the temperature independent relaxation efficiency could be explained by assuming an identical temperature dependence of $W_{32}$ and $W_{31}$. This scenario is considered unlikely due to the significantly different energy of the ${ }^{4} I_{11 / 2} \rightarrow{ }^{4} I_{15 / 2}$ and ${ }^{4} I_{11 / 2} \rightarrow{ }^{4} I_{13 / 2}$ transitions (1.26 and $0.45 \mathrm{eV}$, respectively). Based on these considerations it seems most likely that the decay from the second excited state is dominated by the relaxation to the first excited state $\left(W_{3} \approx W_{32}\right)$, suggesting that the relaxation efficiency $\eta_{32}$ in this material is near-unity.

In summary, luminescence-center-mediated $\mathrm{Er}^{3+}$ excitation is shown to occur with near-equal probability through direct excitation into the first excited state (45\%-50\%) and through excitation into higher levels followed by relaxation to the first excited state $(50 \%-55 \%)$ in the entire temperature range $15-300 \mathrm{~K}$. The observation of a temperatureindependent $\mathrm{Er}^{3+}$ excitation rate and a temperatureindependent fraction of $\mathrm{Er}^{3+}$ excitation events occurring via the second excited state combined with the observed increasing second excited state relaxation rate suggests a constant near-unity relaxation efficiency from the second to the first excited state of $\mathrm{Er}^{3+}$ in low-temperature-annealed Er-doped Si-rich $\mathrm{SiO}_{2}$ in this temperature range. These results indicate that high pump efficiency and stable room temperature operation of devices based on this material are possible.

This work was supported by the National Science Foundation CAREER No. ECCS-0644228.

${ }^{1}$ M. Lipson, J. Lightwave Technol. 23, 4222 (2005).

${ }^{2}$ M. Makarova, V. Sih, J. Warga, R. Li, L. Dal Negro, and J. Vuckovic, Appl. Phys. Lett. 92, 161107 (2008).

${ }^{3}$ L. Dal Negro, R. Li, J. Warga, and S. N. Basu, Appl. Phys. Lett. 92, 181105 (2008).

${ }^{4}$ Q. Lin, T. J. Johnson, R. Perahia, C. P. Michael, and O. J. Painter, Opt. Express 16, 10596 (2008).

${ }^{5}$ T. J. Kippenberg, J. Kalkman, A. Polman, and K. J. Vahala, Phys. Rev. A 74, 051802 (2006).

${ }^{6}$ O. Savchyn, F. R. Ruhge, P. G. Kik, R. M. Todi, K. R. Coffey, H. Nukala, and H. Heinrich, Phys. Rev. B 76, 195419 (2007).

${ }^{7}$ O. Savchyn, P. G. Kik, R. M. Todi, and K. R. Coffey, Phys. Rev. B 77, 205438 (2008).

${ }^{8}$ G. Franzò, S. Boninelli, D. Pacifici, F. Priolo, F. Iacona, and C. Bongiorno, Appl. Phys. Lett. 82, 3871 (2003).

${ }^{9}$ D. Navarro-Urrios, A. Pitanti, N. Daldosso, F. Gourbilleau, R. Rizk, G. Pucker, and L. Pavesi, Appl. Phys. Lett. 92, 051101 (2008).

${ }^{10}$ R. D. Kekatpure and M. L. Brongersma, Nano Lett. 8, 3787 (2008).

${ }^{11}$ R. D. Kekatpure and M. L. Brongersma, Phys. Rev. A 78, 023829 (2008).

${ }^{12}$ O. Savchyn, R. M. Todi, K. R. Coffey, and P. G. Kik, Appl. Phys. Lett. 93, 233120 (2008).

${ }^{13}$ M. Ya. Valakh, V. A. Yukhimchuk, V. Ya. Bratus, A. A. Konchits, P. L. F. Hemment, and T. Komoda, J. Appl. Phys. 85, 168 (1999).

${ }^{14}$ C. B. Layne, W. H. Lowdermilk, and M. J. Weber, Phys. Rev. B 16, 10 (1977).

${ }^{15}$ F. L. Galeener and G. Lucovsky, Phys. Rev. Lett. 37, 1474 (1976)

${ }^{16}$ C. T. Kirk, Phys. Rev. B 38, 1255 (1988).

${ }^{17}$ E. Desurvire, Erbium-doped Fiber Amplifiers: Principles and Applications (Wiley, Hoboken, New Jersey, 2002), Chap. 4, pp. 223-224. 